\title{
Exploring the possibility of predicting human head hair greying from DNA using whole-exome and targeted NGS data
}

Ewelina Pośpiech ${ }^{1 *}$ (D) Magdalena Kukla-Bartoszek ${ }^{1,2}$, Joanna Karłowska-Pik ${ }^{3}$, Piotr Zieliński ${ }^{4}$, Anna Woźniak ${ }^{5}$, Michał Boroń ${ }^{5}$, Michał Dąbrowski ${ }^{6}$, Magdalena Zubańska ${ }^{7}$, Agata Jarosz ${ }^{1}$, Tomasz Grzybowski ${ }^{8}$, Rafał Płoski ${ }^{9}$, Magdalena Spólnicka ${ }^{5}$ and Wojciech Branicki ${ }^{1,5}$

\begin{abstract}
Background: Greying of the hair is an obvious sign of human aging. In addition to age, sex- and ancestry-specific patterns of hair greying are also observed and the progression of greying may be affected by environmental factors. However, little is known about the genetic control of this process. This study aimed to assess the potential of genetic data to predict hair greying in a population of nearly 1000 individuals from Poland.

Results: The study involved whole-exome sequencing followed by targeted analysis of 378 exome-wide and literature-based selected SNPs. For the selection of predictors, the minimum redundancy maximum relevance (mRMRe) method was used, and then two prediction models were developed. The models included age, sex and 13 unique SNPs. Two SNPs of the highest mRMRe score included whole-exome identified KIF1A rs59733750 and previously linked with hair loss FGF5 rs7680591. The model for greying vs. no greying prediction achieved accuracy of cross-validated AUC $=0.873$. In the 3-grade classification cross-validated AUC equalled 0.864 for no greying, 0.791 for mild greying and 0.875 for severe greying. Although these values present fairly accurate prediction, most of the prediction information was brought by age alone. Genetic variants explained $<10 \%$ of hair greying variation and the impact of particular SNPs on prediction accuracy was found to be small.

Conclusions: The rate of changes in human progressive traits shows inter-individual variation, therefore they are perceived as biomarkers of the biological age of the organism. The knowledge on the mechanisms underlying phenotypic aging can be of special interest to the medicine, cosmetics industry and forensics. Our study improves the knowledge on the genetics underlying hair greying processes, presents prototype models for prediction and proves hair greying being genetically a very complex trait. Finally, we propose a four-step approach based on genetic and epigenetic data analysis allowing for i) sex determination; ii) genetic ancestry inference; iii) greyingassociated SNPs assignment and iv) epigenetic age estimation, all needed for a final prediction of greying.
\end{abstract}

Keywords: Head hair greying, Whole-exome sequencing, Targeted next-generation sequencing, Prediction modelling, KIFTA, FGF5

\footnotetext{
* Correspondence: ewelina.pospiech@uj.edu.pl

${ }^{1}$ Malopolska Centre of Biotechnology, Jagiellonian University, Kraków, Poland

Full list of author information is available at the end of the article
}

C The Author(s). 2020 Open Access This article is licensed under a Creative Commons Attribution 4.0 International License, which permits use, sharing, adaptation, distribution and reproduction in any medium or format, as long as you give appropriate credit to the original author(s) and the source, provide a link to the Creative Commons licence, and indicate if changes were made. The images or other third party material in this article are included in the article's Creative Commons licence, unless indicated otherwise in a credit line to the material. If material is not included in the article's Creative Commons licence and your intended use is not permitted by statutory regulation or exceeds the permitted use, you will need to obtain permission directly from the copyright holder. To view a copy of this licence, visit http://creativecommons.org/licenses/by/4.0/. The Creative Commons Public Domain Dedication waiver (http://creativecommons.org/publicdomain/zero/1.0/) applies to the data made available in this article, unless otherwise stated in a credit line to the data. 


\section{Background}

Aging is inherently connected with changes in human appearance. Progressive physical traits include skin aging signs, greying of hair and hair loss and are perceived as biomarkers of individual's aging rate and general health [1-3]. Therefore, there is an increasing interest in understanding the mechanisms underlying the differences in the pace of changes in human appearance and thus the mechanisms of aging processes. This knowledge could find practical application in medicine by boosting the accuracy of assessment of the risk of age-related diseases and in cosmetic industry in order to develop products that could prevent or slow down the clinical signs of phenotypic aging $[1,4,5]$. The studies on age-related physical traits are also useful in forensics and anthropology. Genetic prediction of human appearance based on DNA left at the crime scene or extracted from human remains may speed up the process of human identification [6].

Hair greying is an age-dependent trait and is understood as a progressive loss of pigment from the growing hair shaft. According to the $50-50-50$ rule about $50 \%$ of the population experiences about $50 \%$ of grey hair at the age of 50 years [7]. Under healthy aging conditions, the onset of hair greying in Europeans occurs at the age of $\sim 35$ years, while greying observed under the age of 30 years is usually termed as premature hair greying $[8,9]$. However, the progression of hair greying varies between populations with Africans and Asians showing less grey hair with the onset of hair greying occurring $\sim 10$ years later when comparing to Europeans [10, 11]. Although the cause of hair greying has been extensively studied, it is still poorly understood, appears to be very complex and may involve many different mechanisms [5, 9]. These mechanisms include dysfunction of the follicular melanocytes and thus pigmentary machinery malfunction and defective self-maintenance of the melanocyte stem cells (MSCs) present in the hair bulge [8, 12]. MSCs are activated during hair regeneration, migrate out from the bulge to the hair matrix region and differentiate into pigment producing melanocytes. Therefore, numerous factors involved in a proper regulation of the MSCs maintenance can be involved in the development of hair greying. A factor that seems to contribute to all of the mechanisms leading to hair greying is oxidative stress $[5,9,13]$. Such oxidative toxicity in the hair follicle can be induced by both intrinsic (melanogenesis itself, genetics, hormones, active hair growth) and extrinsic (UV exposure, inflammation, drugs, smoking, obesity, emotional stress, poor nutrition) factors [5, 14-16].

Little is known about genetic predispositions to the development of early greying. Recent analyses conducted by Adhikari and colleagues have shown that hair greying exhibits only $27 \%$ heritability [17] in contrast to previous studies that pointed out the key contribution of genetic factors in hair greying susceptibility ( $90 \%$ heritability) $[2$, 16]. However, it has been revealed that different methods used for heritability measurement in genomewide association studies (GWAS) studies have different accuracies. In particular, the method used by Adhikari et al. (REML implemented in GCTA method) has been shown to consistently underestimate the trait heritability level [18]. The abovementioned group has conducted GWAS on Latin Americans that revealed only one gene, IRF4, being significantly associated with hair greying [17]. IRF4 has been previously associated with various appearance traits including hair colour, freckles and hair loss e.g. [19-22]. IRF4 encodes interferon regulatory factor that interacts with the MITF transcription factor. MITF activates expression of the TYR gene encoding tyrosinase, a key enzyme in melanin synthesis [23]. Importantly, MITF has been also suggested to be engaged in MSCs maintenance through induction of the expression of the $B C L 2$ gene, a key factor in protection against oxidative stress in melanocytes [12, 24]. However, IRF4 rs12203592 was found to explain only a small proportion of the total variation observed in head hair greying [17].

The goal of the current study was to assess the potential of genetic variants to predict head hair greying status in individuals of European descent. The study included a discovery stage which involved the exome-wide association analysis. Additionally, an expanded list of DNA variants previously associated with pigmentation, head hair shape/thickness and head hair loss was also evaluated for their association with head hair greying and their impact on prediction accuracy. Finally, the minimum redundancy maximum relevance (mRMRe) method was used for predictors selection and a set of carefully selected SNPs combined with the information on age and sex was next used to develop the prototype models for head hair greying prediction.

\section{Results}

\section{Characteristics of the study population}

Hair greying was investigated in a set of 998 individuals from Poland aged $\geq 18$ years. Study population included 673 males $(67.4 \%)$ and 325 (32.6\%) females. The mean age of the participants was $30.5 \pm 8.8$. Participants were assessed for grey hair using 6-stage classification (Fig. 1). For 26 samples only binary classification of hair greying was available (greying vs. no greying). Since information on the age of onset of hair greying was not available for all samples or was given only approximately, we did not use this information in the final statistical calculations. As expected, age was found to be significantly correlated with hair greying (Pearson's $r=0.637, P$-value $=2.183 \times$ $10^{-114}$ ). Hair greying was recorded in $14.3 \%$ of 


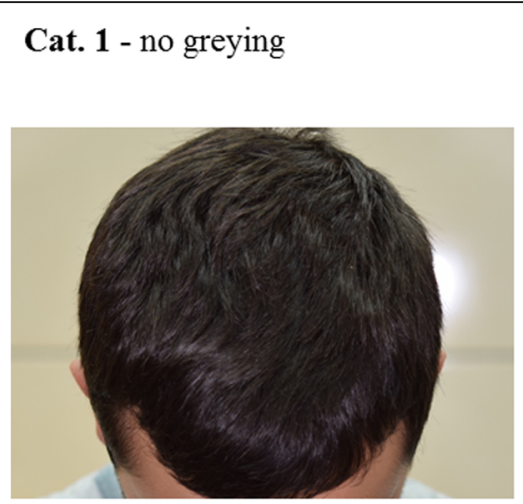

Cat. 4 - significant greying with patches of grey hair

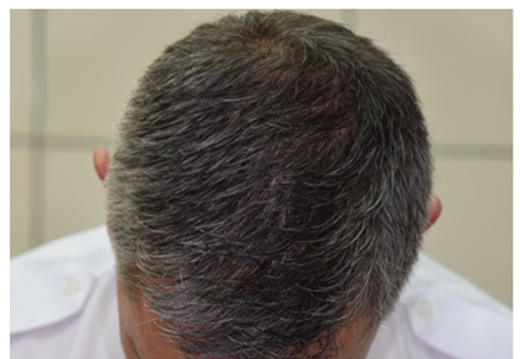

Cat. 2 - predominantly no greying, low number of single grey hair

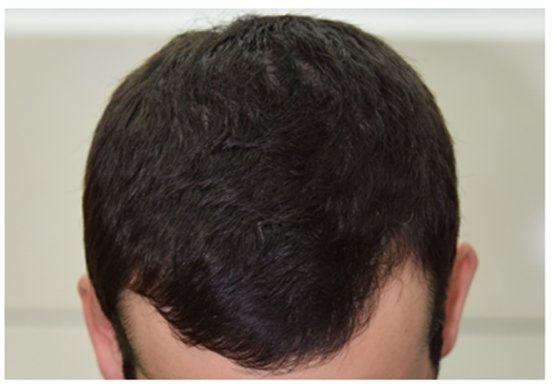

Cat. 5 - predominant greying

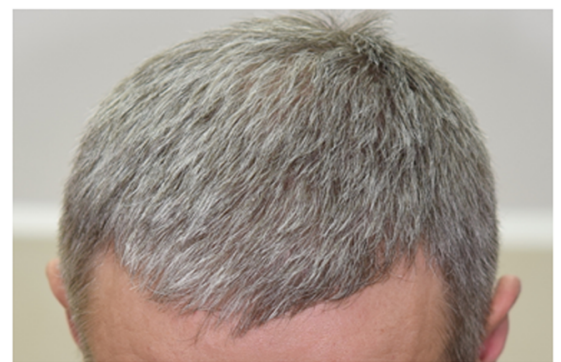

Cat. 3 - higher number of single grey hair (all over the head)

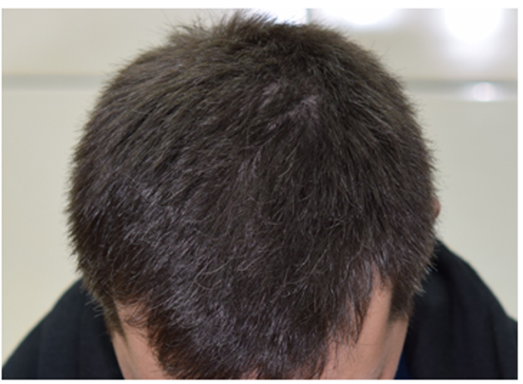

Cat. 6 - totally white hair

Fig. 1 Hair greying 6-stage classification and examples

individuals aged 18-30 and the prevalence of grey hair was noted to be significantly higher in young males when comparing to young females (17.8 and $9.2 \%$, respectively; $x^{2} P$-value $\left.=0.004\right)$. The incidence of grey hair increased to $29.5 \%$ in the group of people aged $18-40$ years and was $84.2 \%$ when people aged $\geq 40$ years were considered. Analysis of head hair feature correlations showed significant and positive correlation of grey hair with dark hair colour (Pearson's $r=0.152, P$-value $\left.=1.432 \times 10^{-6}\right)$ and with hair loss in men (Pearson's $r=0.297, P$-value $=4.400 \times 10^{-15}$ ) but not with hair shape (Pearson's $r=-0.044, P$-value $=0.169$ ). Straight hair was weakly and negatively correlated with grey hair but only when 6 hair greying categories were considered (Pearson's $r=-0.084, P$-value $=0.009$ ).

A set of carefully selected (maintaining an appropriate age distribution and representativeness of particular hair greying categories) 149 samples was used as the discovery cohort for whole-exome sequencing (WES). The remaining 849 samples were used as a replication and prediction modelling cohort for the purpose of i) validation of DNA variants disclosed by WES; ii) validation of literature-based selected SNPs; iii) subsequent prediction model development. The characteristics of the sample cohorts under study are summarized in Supplementary Table 1.

\section{Exome-wide SNP association testing}

WES enriched by regulome sequencing was conducted for 149 individuals with the defined degree of hair greying. After bioinformatic analysis and quality control filtering steps over $77 \mathrm{~K}$ common SNPs $(M A F \geq 5 \%)$ located within targeted regions and meeting the established criteria were extracted and subjected to exome-wide association testing. Regression analyses have revealed association with $P$ value $<5 \times 10^{-4}$ for 50 SNPs. LD-pruning $\left(\mathrm{r}^{2} \leq 0.7\right)$ reduced the list of SNPs to 34 independent signals (Supplementary Tables 2-3). Two SNPs from this list showed nominal association $(P$-value $<0.05)$ in an independent cohort of 849 individuals (Table 1 and Supplementary Table 4). KIF1A rs59733750 (chr2:240780193) was found to be significantly associated with hair greying in all regression analyses with the highest significance achieved in multinomial ordinal logistic regression for 6 hair greying categories $($ MLR6 $)\left(P\right.$-value $\left.=5.473 \times 10^{-4}\right)$. NSMCE1 rs1127228 (chr16:27226789) was found to be significantly associated with 3-stage and 6-stage hair greying classifications with the highest significance obtained in multinomial ordinal logistic regression for 3 hair greying categories $(M L R 3)(P$-value $=0.015)$ (Table 1). 
Table 1 Selection of exome-wide significant $\left(P\right.$-value $\left.<5 \times 10^{-4}\right)$ and replicated $(P$-value $<0.05)$ SNPs associated with hair greying in a discovery and replication cohorts of 149 and 849 individuals from Poland, respectively

\begin{tabular}{|c|c|c|c|c|c|c|c|c|c|}
\hline \multirow[t]{2}{*}{ SNP_ID } & \multirow{2}{*}{$\begin{array}{l}\text { Chr position } \\
\text { GRCh38 }\end{array}$} & \multirow[t]{2}{*}{ Gene } & \multirow[t]{2}{*}{ fMA } & \multicolumn{2}{|l|}{ BLR } & \multicolumn{2}{|l|}{ MLR3 } & \multicolumn{2}{|l|}{ MLR6 } \\
\hline & & & & beta & $P$-value ${ }^{a}$ & beta & $P$-value ${ }^{a}$ & beta & $P$-value ${ }^{a}$ \\
\hline \multicolumn{10}{|c|}{ Discovery cohort $(N=149) ; P$-value $<5 \times 10^{-4}$} \\
\hline rs59733750 & 2:240780193 & KIFIA & G 0.144 & -1.782 & 0.013 & -1.787 & 0.002 & -1.861 & $2.798 \times 10^{-4}$ \\
\hline rs1127228 & 16:27226789 & NSMCE1 & T 0.362 & -1.360 & 0.005 & -1.452 & $4.312 \times 10^{-4}$ & -1.404 & $1.227 \times 10^{-4}$ \\
\hline \multicolumn{10}{|c|}{ Replication cohort $(N=849) ; P$-value $<0.05$} \\
\hline rs59733750 & 2:240780193 & KIFIA & G 0.163 & -0.484 & 0.007 & -0.541 & 0.002 & -0.569 & $5.473 \times 10^{-4}$ \\
\hline rs1127228 & $16: 27226789$ & NSMCE1 & Т 0.347 & -0.241 & 0.079 & -0.314 & 0.015 & -0.283 & 0.022 \\
\hline
\end{tabular}

BLR, binomial logistic regression; MLR3, multinomial ordinal logistic regression for 3 hair greying categories; MLR6, multinomial ordinal logistic regression for 6 hair greying categories; MA, minor allele; fMA, frequency of minor allele

${ }^{\text {a }}$ Results adjusted for age, sex and hair colour

\section{Association testing for literature-based selected candidate hair greying SNPs}

IRF4 rs12203592 (chr6:396321) was the only SNP discovered by Adhikari and colleagues of genome-wide significance in the only one genome-wide association study conducted so far for hair greying [17]. In the current study we replicated the effect of IRF4 rs12203592 and showed it to be significantly associated with heir greying in a cohort of 849 samples (Table 2). In an univariate binomial regression analysis conducted for greying vs. no greying the minor $\mathrm{T}$ allele ( $\mathrm{fMA}=0.08$ ) was found to increase the odds of grey hair by a factor of $2.0(95 \% \mathrm{CI}=$ $1.3-3.2$, age and sex adjusted $P$-value $=0.003)$. From the Nagelkerke $R^{2}$ statistic, this SNP explains $0.9 \%$ of the total variation observed in hair greying. To visualize the effect of IRF4 rs12203592 on hair greying development CHAID tree analysis was conducted. As expected, age was at the top of the tree emphasizing its dominant role in hair greying formation (Fig. 2). Individuals $\leq 30$ years old have only $14.9 \%$ probability of grey hair. IRF4 rs12203592 $\mathrm{T}$ allele was found to impact grey hair occurrence in this youngest group of individuals increasing the probability of grey hair in CT/TT-rs12203592 individuals by $\sim 15$ p.p. (to a final probability of $27.3 \%$ ) when comparing to CC-rs12203592 carriers.

Although IRF4 rs12203592 was the only SNP that reached genome-wide significance in a study conducted by Adhikari et al., several suggestive associations have been also revealed for additional 7 loci. Besides IRF4 rs12203592 we replicated the effect of $M R O H 2 A$ rs2361506 (chr2:233830694) $(\mathrm{OR}=1.4,95 \% \mathrm{CI}=1.1-1.9$, age and sex adjusted $P$-value $=0.008$ ) (Table 2). Age, IRF4 rs12203592 and $M R O H 2 A$ rs2361506 altogether were found to explain $49.1 \%$ of variation observed in hair greying with $47.5 \%$ of variation explained by age and the remaining $1.6 \%$ explained by IRF4 and $\mathrm{MROH} 2 \mathrm{~A}$.

Because there are indications that the genetics underlying the various head characteristics may overlap to some extent, we evaluated the relationship to hair greying for additional list of 336 SNPs previously associated with hair colour / pigmentation, hair loss, shape and

Table 2 Validation of SNPs associated with hair greying by Adhikari et al. (2016) in a replication cohort of 849 individuals from Poland

\begin{tabular}{|c|c|c|c|c|c|c|c|c|c|}
\hline \multirow[t]{2}{*}{ SNP_ID } & \multirow{2}{*}{$\begin{array}{l}\text { Chr position } \\
\text { GRCh38 }\end{array}$} & \multirow[t]{2}{*}{ Gene } & \multirow[t]{2}{*}{ fMA } & \multicolumn{2}{|l|}{ BLR } & \multicolumn{2}{|l|}{ MLR3 } & \multicolumn{2}{|l|}{ MLR6 } \\
\hline & & & & beta & $P$-value ${ }^{a}$ & beta & $P$-value ${ }^{\mathrm{a}}$ & beta & $P$-value ${ }^{*}$ \\
\hline rs12203592 & chr6:396321 & IRF4 & T 0.08 & 0.700 & 0.003 & 0.669 & 0.002 & 0.780 & $1.121 \times 10^{-4}$ \\
\hline rs2361506 & chr2:233830694 & $M R O H 2 A$ & Т 0.37 & 0.360 & 0.008 & 0.220 & 0.077 & 0.156 & 0.183 \\
\hline rs2085601 & chr4:88974793 & FAM13A & C 0.31 & -0.068 & 0.630 & -0.099 & 0.460 & -0.056 & 0.658 \\
\hline rs7009516 & chr8:24351334 & ADAM28 & G 0.46 & 0.036 & 0.785 & -0.099 & 0.420 & -0.107 & 0.361 \\
\hline rs1912702 & chr11:79462038 & MIR708; TENM4 & T 0.37 & 0.029 & 0.823 & -0.022 & 0.854 & -0.012 & 0.917 \\
\hline rs11621135 & chr14:71192892 & PCNX; LOC145474; SNORD56B & A 0.44 & 0.028 & 0.829 & 0.065 & 0.588 & 0.031 & 0.786 \\
\hline rs281229 & chr15:47426258 & SEMA6D & T 0.00 & $-b^{b}$ & $-\mathrm{b}$ & $-\mathrm{b}$ & $-\mathrm{b}$ & $-^{* *}$ & $-^{* *}$ \\
\hline rs1005241 & chr22:47291868 & LOC101927722; TBC1D22A & C 0.45 & -0.153 & 0.252 & -0.097 & 0.441 & -0.075 & 0.530 \\
\hline
\end{tabular}

Significant results $(P$-value $<0.05)$ are marked with bold

BLR, binomial logistic regression; MLR3, multinomial ordinal logistic regression for 3 hair greying categories; MLR6, multinomial ordinal logistic regression for 6 hair greying categories; MA, minor allele; $\mathrm{AMA}$, frequency of minor allele

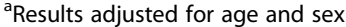

${ }^{\mathrm{b}}$ Monomorphic SNP 


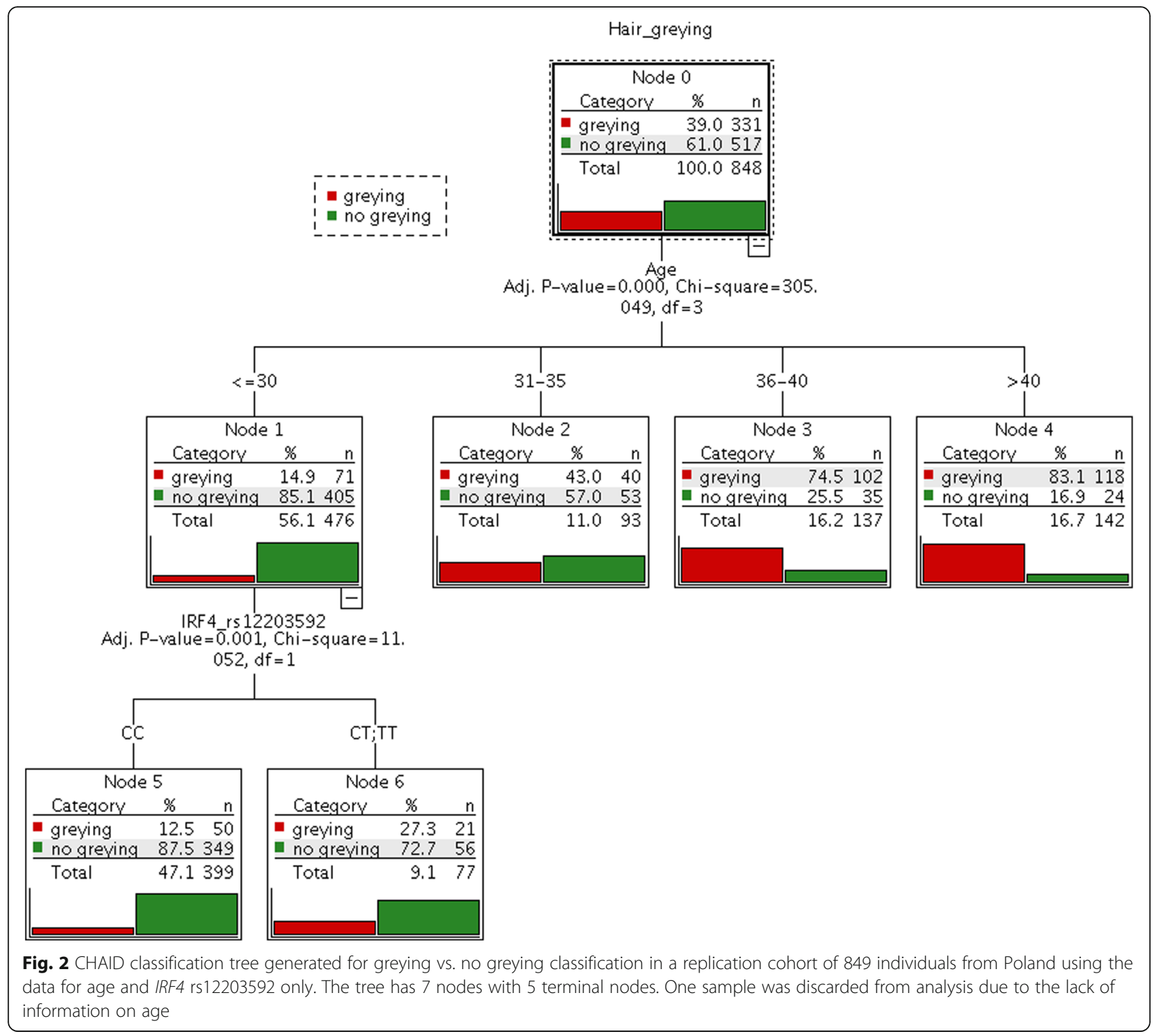

thickness. Out of these SNPs, positive signal of association, considering $P$-value $<0.05$ as statistically significant and achieved in at least one of regression tests, was observed for 35 SNPs from 24 loci; ERRFI1/ SLC45A1, TCHH/RPTN, TEX41, HOXD-AS2/HOXD3, LOC391485, FGF5, EBF1, IRF4, SNX13, BRINP1, 10p14, GATA3, GRID1, 11q24.2, KRT71, P2RY5, OCA2, HERC2, 16q24.1, DPEP1, DEF8, APCDD1, PTK6 and RUNX1. Of the above, 8 loci (9 SNPs) were previously associated with hair loss, 8 loci (15 SNPs) were previously associated with pigmentation and the remaining 8 (11 SNPs) were linked to head hair shape (Supplementary Table 5). The highest significance $(P$-value $<0.01)$ was noted for FGF5 rs7680591 (chr4:80276795) with $P$-value $=0.003$ and P-value $=0.004$ in MLR3 and MLR6 analyses, respectively and DPEP1 rs164741 (chr16:89625890) with
$P$-value $=0.004$ and $P$-value $=0.007$ in binomial logistic regression (BLR) and MLR3 analyses, respectively. These two SNPs were previously associated with hair loss and pigmentation, respectively, both explaining less than $1 \%$ of the total variation observed in hair greying in the studied population.

\section{Prediction modelling analyses}

To increase the rate of success in selection of the most relevant set of SNP predictors we applied the mRMRe model selection method allowing simultaneous analysis of large sets of SNPs. SNPs were analysed in a 849sample cohort, ranked according to the mRMRe score and the top 30 variants were extracted for both hair greying classifications (Supplementary Fig. 1 and Supplementary Table 6) and pruned based on analysis of scree 


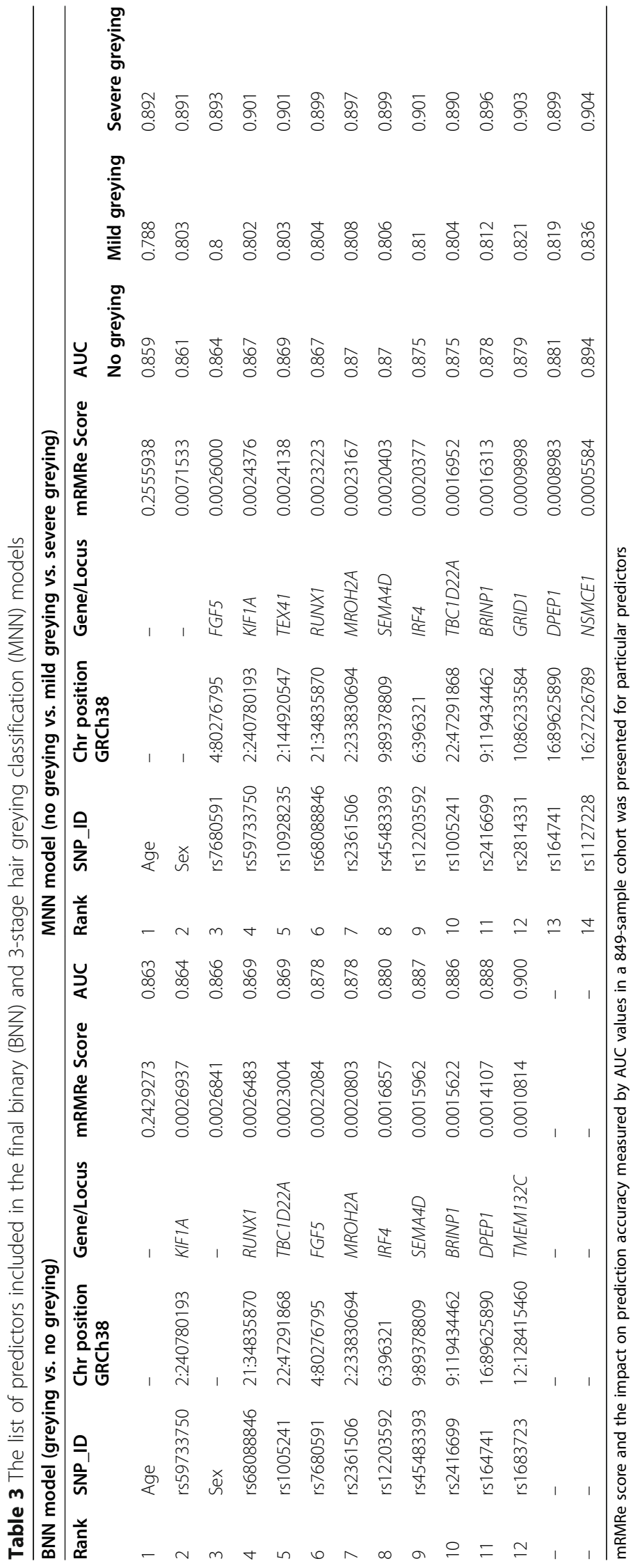


plots (Supplementary Fig. 2). This analysis allowed preselection of 13 predictors for binomial hair greying classification (age + sex + 11 SNPs) and 16 for 3-stage classification (age + sex +14 SNPs). Pre-selected variables were then assessed for their impact on prediction accuracy based on AUC values. This analysis led to a final list of 12 (age + sex + 10 SNPs) and 14 (age + sex + 12 SNPs) predictors included in the final binary neural network $(\mathrm{BNN})$ and multi-class neural network $(\mathrm{MNN})$ prediction models, respectively (Table 3 ). The workflow for the final selection of predictors is summarized on Supplementary Fig. 1. For both hair greying classifications, the highest mRMRe score was attributed to age. Age itself was found to explain Nagelkerke $R^{2} 47.5 \%$ of the total variation observed in hair greying defined in a binary way $(45.5 \%$ for hair 3 -stage classification) and ensures the accuracy of head hair greying prediction at the level of $\mathrm{AUC}=0.863$ for greying vs. no greying and $\mathrm{AUC}=$ 0.859 for no greying, $\mathrm{AUC}=0.788$ for mild greying, AUC $=0.892$ for severe greying in 3-stage classification (Table 3). Sex was ranked at the third position in BNN and at the second position in MNN but in contrast to age, sex explains significantly smaller proportion of the total variance observed in hair greying $(5.5 \%$ for $\mathrm{BNN}$ and $7.3 \%$ for $\mathrm{MNN}$ ) and its impact on prediction accuracy was also found to be lower. Thirteen unique SNPs were included in BNN and/or MNN models with 9 SNPs overlapping between both sets of predictors. These 13 DNA variants included 4 exome-wide identified SNPs (KIF1A rs59733750, NSMCE1 rs1127228, SEMA4D rs45483393, TMEM132C rs1683723), 3 SNPs associated with hair greying in Adhikari et al. (IRF4 rs12203592, MROH2A rs2361506, TBC1D22A rs1005241), 5 SNPs previously associated with hair loss (FGF5 rs7680591, TEX41 rs10928235, RUNX1 rs68088846, BRINP1 rs2416699, GRID1 rs2814331) and 1 SNP previously linked with pigmentation (DPEP1 rs164741). Ten out of 13 SNPs were found to achieve nominally significant association in regression tests (Tables 1 and 2, Supplementary Table 5), the remaining 3 SNPs included two SNPs (SEMA4D rs45483393 and TMEM132C rs1683723) identified with exome sequencing but not replicated in a set of 849 samples and one SNP (TBC1D22A rs1005241) with suggestive association in Adhikari et al. [17]. Among all the SNPs included in the models the highest
mRMRe score was attributed to exome-wide identified KIF1 rs59733750 ( $2^{\text {nd }}$ place in $\mathrm{BNN}$ and $4^{\text {rd }}$ place in $\mathrm{MNN}$ ) and previously linked with hair loss FGF5 rs7680591 ( $3^{\text {rd }}$ place in MNN model and $6^{\text {th }}$ place in $\mathrm{BNN})$. Genetic variants were found to explain only a very small percentage of total variance in head hair greying. Altogether, 10 SNPs from BNN model and 12 SNPs from MNN explains 7.3\% (total variance explained by age + sex + SNPs $=52.7 \%$ ) and 9\% (total variance explained by age + sex + SNPs $=52.4 \%$ ), respectively. Therefore, their impact on prediction accuracy was found to be small with AUC change $<0.02$ for individual SNPs (Table 3). Final models built on 849-sample set and including information on age, sex and DNA variation were found to predict hair greying status with accuracy of $\mathrm{AUC}=0.900$ for greying vs. no greying (increase by 0.037 when comparing to age-based model) and AUC $=0.894$ for no greying (increase by $0.035 \mathrm{com}$ paring to age-based model), $\mathrm{AUC}=0.836$ for mild greying (increase by 0.048 ) and $\mathrm{AUC}=0.904$ (increase by 0.012 ) for severe greying in 3-stage classification.

The accuracy of prediction of the final $\mathrm{BNN}$ and MNN hair greying models was further assessed by 10 fold cross-validation (CV). The cross-validated AUC values obtained were 0.873 for $\mathrm{BNN}$ and $0.864,0.791$ and 0.875 for no greying, mild greying and severe greying categories in MNN, respectively (Table 4). Sensitivity achieved for BNN equalled 0.734; out of 331 individuals with hair greying symptoms for 243 of them prediction was correct. Specificity was higher and reached 0.854; 442 individuals out of 518 individuals without any signs of hair greying were correctly assigned to no greying category. PPV value reached 0.762 which means that in 319 cases in which hair were classified as greying, for 243 of them prediction result was correct. NPV value at the level of 0.834 means that out of 530 no hair greying classifications in $83.4 \%$ of them prediction was correct and individuals indeed did not show any signs of hair greying. In case of MNN model, the highest sensitivity at the level of 0.886 was achieved for no greying category, the sensitivity for mild greying was 0.589 while the lowest value was gained for severe greying with only $7.7 \%$ of severe greying cases correctly predicted. However, for severe greying category the highest specificity was obtained at the level of 0.997 . The specificity for no greying

Table 4 Final accuracy estimates of the BNN and MNN models for hair greying prediction designated in a 849-sample cohort using 10-fold cross-validation procedure

\begin{tabular}{lllllll}
\hline Model & & AUC & Sensitivity & Specificity & PPV & NPV \\
\hline 12-variable BNN & greying vs. no greying & 0.873 & $0.734(243 / 331)$ & $0.853(442 / 518)$ & $0.762(243 / 319)$ & $0.834(442 / 530)$ \\
14-variable MNN & no greying & 0.864 & $0.886(459 / 518)$ & $0.643(196 / 305)$ & $0.808(459 / 568)$ & $0.769(196 / 255)$ \\
& mild greying & 0.791 & $0.589(149 / 253)$ & $0.821(468 / 570)$ & $0.594(149 / 251)$ & $0.818(468 / 572)$ \\
& severe greying & 0.875 & $0.077(4 / 52)$ & $0.997(769 / 771)$ & $0.667(2 / 6)$ & $0.941(769 / 817)$ \\
\hline
\end{tabular}




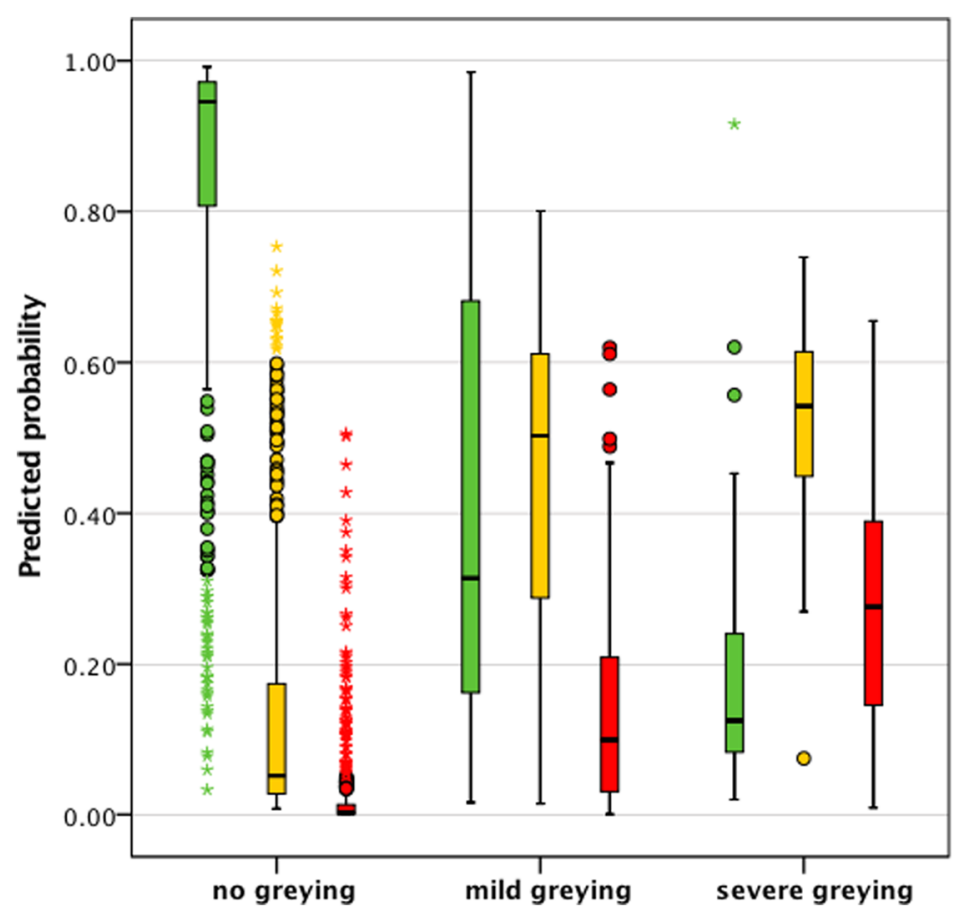

Fig. 3 The distribution of hair greying predicted probabilities generated with MNN model

and mild greying equalled 0.643 and 0.821 , respectively (Table 4).

Low value of sensitivity observed for severe greying category means that most of the samples of severe greying (42/52) were classified as mild greying which indicates problems with differentiation between mild and severe greying categories. This is reflected by high probabilities for mild greying category generated for samples in severe greying category (Fig. 3). However, at the same time, an analysis of the distribution of probabilities generated with MNN model shows expected increase in the probability of severe greying when moving from no greying to severe greying category. The mean probability for severe greying category in mild greying category equalled $11.4 \pm 10.5 \%$ while for individuals in severe greying category increased to $20.7 \pm 13.6 \%\left(P\right.$-value $\left.=9.62 \times 10^{-8}\right)$.

The total number of correct predictions achieved with BNN model equalled 684/848 (80.7\%) and was higher when comparing to the model built using information on age only (677/848) (Table 5). As the goal of the study was to evaluate if information on SNPs can improve accuracy of hair greying predictions, we focused on individuals i) young ( $(\leq 30$ years old $)$ and greying, $N=71$ and ii) older ( $\geq 40$ years old) and non-greying, $N=29$ where age itself is non-informative $(0 \%$ of correct predictions based on sole information from age, Table 5). This analysis showed that information on SNPs included in the models allowed proper recognition of $6(8.5 \%)(\mathrm{BNN})$ or 3 (4.4\%) $(\mathrm{MNN})$ young and greying individuals and $2(6.9 \%)$ old and no greying individuals when using MNN model.

\section{Discussion}

\section{The etiology of hair greying}

Hair greying occurs as people age, but its progression is influenced by many different factors including sex,

Table 5 Success rate in prediction of hair greying status in a total 849-sample set and in two extreme phenotypic groups; i) $\leq 30$ years old and greying, ii) $\geq 40$ years old and no greying

\begin{tabular}{llll}
\hline Correct predictions & Total $N$ & Young $(\leq 30$ y.o.) and greying & Old $(\geq 40$ y.o.) and no greying \\
\hline Age-based BNN model & $677 / 848^{\mathrm{a}}(79.8 \%)$ & $0 / 71(0.0 \%)$ & $0 / 29(0.0 \%)$ \\
12-variable BNN model & $684 / 848^{\mathrm{a}}(80.7 \%)$ & $6 / 71(8.5 \%)$ & $0 / 29(0.0 \%)$ \\
Age-based MNN model & $609 / 822^{\mathrm{b}}(74.1 \%)$ & $0 / 68(0.0 \%)$ & $0 / 29(0.0 \%)$ \\
14-variable MNN model & $611 / 822^{\mathrm{b}}(74.3 \%)$ & $3 / 68(4.4 \%)$ & $2 / 29(6.9 \%)$ \\
\hline
\end{tabular}

${ }^{a}$ One sample was discarded from BNN analyses due to the lack of information on age

${ }^{b}$ One sample was discarded from MNN analyses due to the lack of information on age and 26 additional samples were omitted due to the availability of information on binary status of hair greying only 
biogeographic ancestry, genetic predispositions and the impact of environment/lifestyle [5, 10, 16]. The average time before a first grey hair appears was estimated at age $~ 35$ years in Europeans [11]. In our study $\sim 14 \%$ of people at age below 30 years had grey hair and therefore were diagnosed with premature hair greying. The incidence of grey hair was found to be higher in males than females which is concordant with previous reports [11]. Furthermore, our study showed that dark-haired individuals harbor significantly more grey hair than the lighthaired individuals which is in line with previous report [11]. Although the etiology of hair greying has been widely studied, it is still not fully understood. The image that arises from the current research indicates multifactorial background of hair greying with a wide range of different factors, different mechanisms and molecular pathways included $[5,9]$. Hair greying comes along with many diseases including among others Waardenburg syndrome, spastic paraplegia, pernicious anemia and progeria (Hutchinson-Gilford and Werner syndrome) [25-28]. The genetic predisposition to the development of premature greying of hair has been recognized only to a very limited extent. Hair greying appears to be a polygenic trait, with a large number of genes involved with small or medium effect sizes. In a GWA study conducted in 2016 on a large sample of admixed Latin Americans, a single IRF4 gene reached genome-wide significance. rs12203592 in IRF4 was shown to explain merely $\sim 8 \%$ of the total hair greying variation [17]. In the current research we have confirmed the effect of association of IRF4 gene with hair greying in a Polish population and to the best of our knowledge this is the first study replicating the association disclosed by Adhikari et al. [17]. However, the proportion of the variation explained by IRF4 rs12203592 in our study was found to be even smaller $(\sim 1 \%)$. IRF4 rs12203592 was included in both models developed in our study but surprisingly was ranked low by mRMRe, at 8th and 9th positions in BNN and MNN models, respectively. Although IRF4 rs12203592 was the only SNP that achieved GWAsignificance, Adhikari et al. also reported suggestive associations for other 7 SNPs [17]. In univariate tests we replicated association of $M R O H 2 A$ rs 2361506 . This SNP was included in both models (ranked at $7^{\text {th }}$ position).

\section{Prediction modelling strategy}

Although the human genome project provided impressive insights into the genetic architecture of many complex traits, large proportion of heritability of these phenotypes still remains unexplained [29]. Recent studies have demonstrated that accurate prediction of human complex traits may require a change in prediction modelling strategy and inclusion of large number of SNPs selected excluding the association criterion [30,
31]. This strategy gives the chance to reduce the problem of missing heritability that affects genetic prediction $[29,31,32]$. It has been shown that SNPs that show no signs of association in single tests can still improve prediction accuracy (e.g. [33]). Therefore, to increase the rate of success in identification of the relevant predictors for head hair greying: i) the significance threshold was lowered and considered $P$-value of $5 \times 10^{-4}$ as significant in exome-wide association study; ii) the list of candidate DNA variants was expanded by analysis of SNPs previously linked with different head hair features; iii) mRMRe method known to outperform classical approaches in terms of predictors selection was employed (mRMRe does not require SNPs to show significance in single tests). This approach allowed us to develop prototype models for head hair greying prediction that consider information on age, sex and DNA variation.

\section{Genetic component in the hair greying prediction models} The genetic component was found to explain merely $<10 \%$ of hair greying variation in the studied population which is substantially lower than the variation explained by age itself ( $>45 \%)$. Consequently, the impact of particular SNPs on prediction accuracy was small. The list of genes implicated in hair greying phenotype include loci previously linked with smoking status (KIF1A, RUNX1, IRF4, BRINP1, TEX41, GRID1), BMI/obesity (GRID1, TEX41, SEMA4D, TBC1D22A, RUNX1), bone mineral density (RUNX1, TBC1D22A, TEX41), cardiovascular diseases (FGF5, MROH2A, DPEP1, GRID1) and immunology (RUNX1, TBC1D22A, IRF4, TMEM132C, GRID1). Importantly, all the above mentioned factors (particularly smoking) were linked with hair greying syndrome in previous research and therefore the link between these genes and greying seems to be justified [16, 33-36]. Three genes have been previously linked with ageing effects, that is longevity (TBC1D22A), skin aging (IRF4) and DNA methylation aging (RUNX1) [37-39]. Importantly, agerelated changes in DNA methylation can stop melanocyte stem cells growth and thus potentially affect hair greying development [40]. It is also clear that variation in nonprotein coding regions contributes to the phenotype. Of the 13 predictor SNPs in our final hair greying models, only two (rs1683723, rs1127228) affect protein sequence, whereas four (rs12203592, rs68088846, rs45483393, rs1127228) are located in known regulatory regions (enhancer, promoters, a CTCF binding site) [41].

\section{Novel genes associated with hair greying}

Exome-wide association analysis and replication identified two novel DNA variants rs59733750 in KIF1A and rs1127228 in NSMCE1 to be associated with hair greying. Of all SNPs included in the BNN model, rs59733750 in KIF1A received the highest mRMRe score 
(2nd position in the model). KIF1A (2q37.3) encodes a member of the kinesin family proteins that are responsible for the anterograde transport of synaptic-vesicle precursors along axons [42]. Mutations in KIF1A have been previously associated with disorders like spastic paraplegia 30 and hereditary neuropathy $[42,43]$. Interestingly, specific types of spastic paraplegia disorders have been shown to be linked with pigmentary abnormalities, including premature hair greying and also associated with prematurely aged facial appearance $[26,44]$. Noteworthy, neuropathy is the most common complication of diabetes which has been shown to alter expression of KIF1A and can lead to hair follicle damage [4547]. It will be interesting to elucidate the role of KIF1A in molecular etiology behind hair greying. The analysis of genetic variation associated with various diseases can be controversial in forensic genetics for bioethical reasons. However, because pleiotropy is so common, it would be impossible to predict natural phenotypes avoiding genes involved in determination of pathological phenotypes. The penetrance of individual SNP variants is usually low and they altogether can only explain a small fraction of the predisposition to the disease [48] but the inclusion of disease-related genes in models for the genetic prediction of human appearance traits seems inevitable. This topic is currently widely discussed by forensic geneticists and bioethicians.

The second exome-wide identified locus, NSMCE1 (16p12.1), was found to improve the accuracy of hair greying prediction in MNN model (ranked $14^{\text {th }}$ ). The NSMCE1 protein is a RING-type zinc finger-containing E3 ubiquitin ligase which belongs to the structural maintenance of chromosomes (SMC) proteins and is involved in the maintenance of genome integrity, DNA damage response and DNA repair [49]. Hair follicles are exposed to high levels of oxidative stress and the resulting DNA damage. Therefore, defects in DNA repair systems are considered as the important contributors to hair greying development $[5,9,13]$. The final models for hair greying included two additional exome-wide selected loci, SEMA4D rs45483393 and TMEM132C rs1683723 that were among the top mRMRe scored variables and were found to positively affect the accuracy of prediction even though these loci did not achieve statistical significance in single tests conducted in a replication cohort. SEMA4D (9q22.2) encodes semaphoring 4D protein that is a cell surface receptor for PLXNB1 and PLXNB2 and plays a role in axon guidance, immune response, tissue development, cell migration, cell-cell signaling and skin healing process [50]. It is noteworthy that rs 281229 in SEMA6D (15q21.1), which is another member of the semaphoring family, has shown a suggestive association with hair greying in a study conducted by Adhikari et al. [17] but this DNA variant was monomorphic in our population. TMEM132C (12q24.32) encodes transmembrane protein $132 \mathrm{C}$. Transmembrane proteins (TMEM) are components of various cell membranes and play important physiological functions although the biological meaning of particular proteins remains mostly unknown [51]. TMEM proteins were previously implicated in hair shape determination [52].

\section{Pleiotropic effects in head hair features}

Aging of hair follicle is manifested by greying of the hair and hair loss, with both phenotypes being linked to each other [13, 17]. This correlation was also observed in our study. Out of 13 SNPs included in our predictive models for hair greying, 6 (FGF5 rs7680591, RUNX1 rs68088846, IRF4 rs12203592, BRINP1 rs2416699, TEX41 rs10928235, GRID1 rs2814331) were previously associated with male pattern baldness (MPB) [17, 21, 53, 54]. All 6 SNPs showed association with hair greying in our population. The highest significance was noted for IRF4 rs12203592 and FGF5 rs7680591. The FGF5 gene (4q21.21) encodes Fibroblast Growth Factor 5. FGF proteins possess important functions in the regulation of cell growth and are engaged in a broad range of biological processes. FGF proteins have been suggested to be crucial regulators of hair growth [e.g. 15]. Mutations in FGF5 have been linked with trichomegaly, a pathological condition involving abnormally long eyelashes [55]. The role of FGF5 polymorphism in the development of MPB has recently been discovered in two large GWAS studies [21, 54]. Its importance for hair greying also seems possible because active hair growth, which leads to oxidative stress, has been proposed as one of the possible hypotheses for hair greying [15]. Indeed, gene expression of FGF5 was downregulated in grey hair compared to black hair [15]. In our study, rs7680591 in FGF5 was the highest-ranked DNA variant in the MNN model thus highlighting its role in hair greying prediction.

\section{Prediction of hair greying in practice}

Prediction of hair greying status may have a practical value. In forensics, information about hair greying can be used for intelligence purposes [6]. As of yet, first predictive models for hair colour, hair loss and hair shape have been reported [56-60] but there are no studies examining the capabilities of head hair greying prediction. Although the prototype models that have been developed in the current research predicted hair greying status with very high accuracies of $\mathrm{CV}$ AUC $=0.873$ for greying vs. no greying and $\mathrm{CV}$ AUC $=0.864$ for no greying, $\mathrm{CV}$ AUC $=0.791$ for mild greying and $\mathrm{CV}$ AUC = 0.875 for severe greying, the contribution of genetic predictor was very small. Due to the small effects sizes 
attributed to particular DNA variants only 2 or 3 hair greying categories were considered in the final statistical analyses. Larger studies should identify additional predictors that will allow better resolution in the future. It is worth noting that in forensics, the greatest practical significance will be predicting hair greying in extreme age categories. We have shown that the genetic component could correctly predict hair greying status in up to $\sim 9 \%$ of individuals included in extreme phenotype groups. In addition to information about the age of a person, the prediction of hair greying also depends on additional factors, e.g. ancestry and environment. Recent years have proved that aging is associated with widespread changes in genome-wide DNA methylation $[61,62]$. The observed differences in aging rates are in $\sim 30-40 \%$ heritable [63] but can be also influenced by the environment. It might be therefore anticipated that DNA methylation plays a mediating role between the environment and hair greying and that epigenetic age reflecting biological age of a person will be a significant predictor of grey hair, replacing chronological age in the models and at the same time accounting for environmental effects on hair greying. This hypothesis should be evaluated in the future.

Because our predictive models were developed using data for a Polish cohort, further research is needed to confirm their usefulness in other European and worldwide populations. The analysis of the 1000 Genomes data shows significant differences in minor allele frequencies for substantial number of SNPs (Supplementary Fig. 3 A-M). As both the age of onset and the rate of hair greying are linked to ancestry, with generally lower incidence of grey hair observed in African and Asian subjects $[10,11]$, it seems possible that genetic background will also differ to some degree. This is the case with hair loss for which inter-population differences are well described with the lowest incidence observed in Africans and later onset in Asians [57, 64]. To initially evaluate the performance of our SNPs in different populations we have applied our BNN model to 1000 Genome samples (extracted from ensembl.org) and analysed the distribution of hair greying probabilities. Although the phenotypes are not available for 1000 Genome samples we have generally observed significantly lower prediction probability values for grey hair in Africans (Fig. 4). The mean probability for grey hair in Europeans was estimated at $0.38 \pm 0.19$ while for Africans it amounted to $0.23 \pm 0.17\left(P\right.$-value $\left.=5.092 \times 10^{-40}\right)$. This outcome may result from substantial differences in allele frequencies between these two populations for a significant number of SNPs, including the highly rated in our models FGF5 rs7680591 and KIF1A rs59733750 (Supplementary Fig. 3H and 3L).

Prediction of hair greying status solely based on genetic information is currently impossible and, as with

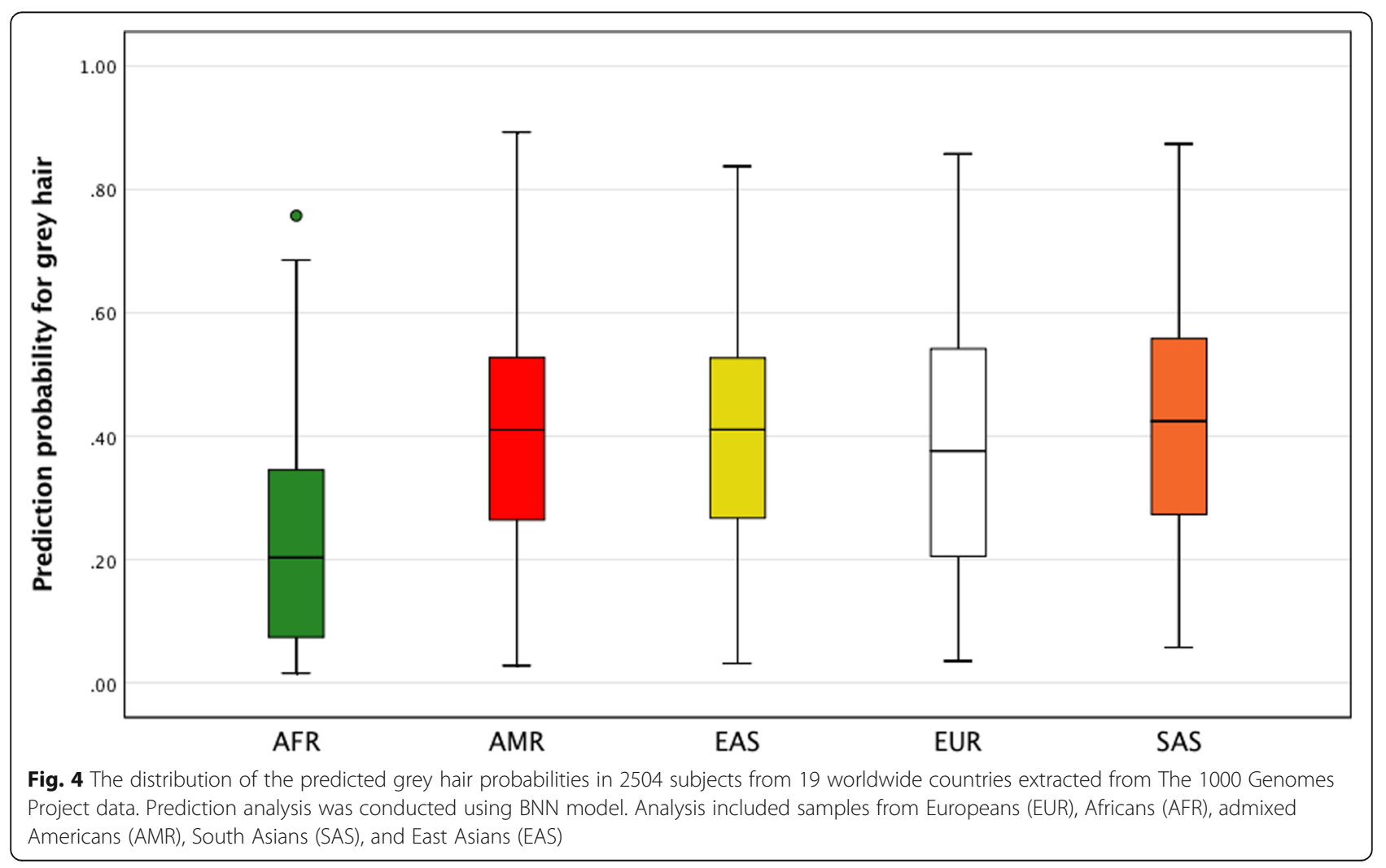


Proposed workflow for hair greying prediction

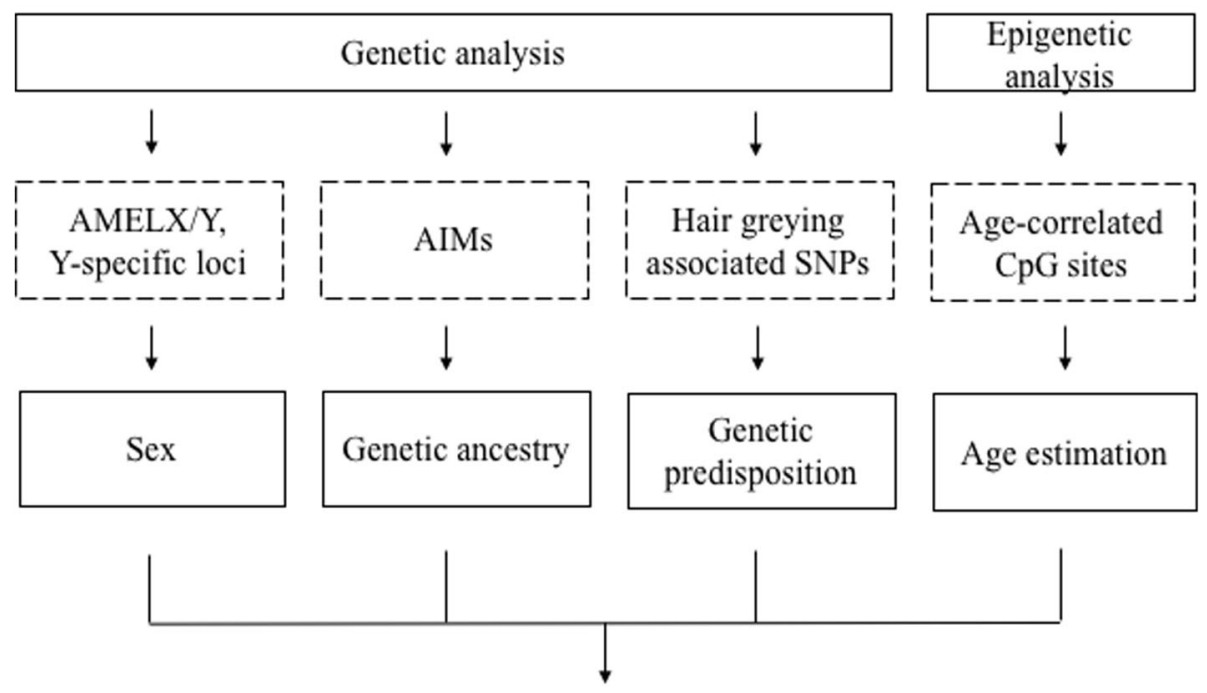

Hair greying
prediction

Fig. 5 Proposed workflow for hair greying prediction based on genetic data and DNA methylation

other progressive human traits, should be accompanied by the estimation of a person's age. It seems that a predictive algorithm based on genetic and epigenetic data may be practical in forensic investigations. Systematic approach that we propose should include: i) sex determination (typically done during standard STR profiling in forensic investigations via analysis of STR markers of Amelogenin gene on chromosomes $\mathrm{X} / \mathrm{Y}$ ); ii) genetic ancestry inference (through analysis of Ancestry Informative Markers (AIM-SNPs and/or AIM-Indels); iii) determination of genetic predispositions to develop hair greying through analysis of greying associated SNPs and iv) epigenetic age estimation (Fig. 5). Analogical solution was proposed to predict facial features. Information about genomic ancestry and sex was used to create a base-face which is next supplemented by genetic information on 24 facial variation associated SNPs [65]. Information on biogeographic ancestry (BGA) can still help predicting various appearance traits. This can complicate the implementation of predictive methods in forensics in those countries where DNA-based BGA inference is restricted by law [66], but cannot change until we fully understand the genetics of the traits, we do not identify all genes and functional variants and their interactions. In case of hair greying, there are clear indications in the literature that the age of onset and the rate of hair greying vary between populations. Future research will show if this is due to differences in allele frequency, different genetic basis and/or epistasis.

\section{Conclusions}

To improve our understanding on the role of DNA variation in hair greying development we have conducted a novel study that enrolled 998 individuals from Poland that were carefully phenotyped for various head hair phenotypes. We have disclosed two novel DNA variants that were selected in a whole-exome analysis conducted in a discovery cohort and successfully validated in a replication cohort, namely KIF1A rs59733750 and NSMCE1 rs1127228. Moreover, we have replicated the association of IRF4 rs12203592 and MROH2A rs2361506 disclosed previously in a GWA study of Adhikari et al. (2016). We have also showed positive signal of association for 35 SNPs from 24 loci previously linked with pigmentation, hair loss and hair shape thus providing another evidence supporting hypothesis that the genetics underlying these characteristics is to some degree overlapping. The prototype models developed for 2- or 3-grade hair greying classification include information on age, sex and DNA variation within 13 unique SNPs, of which 3 variants showed no association in univariate regression tests. The developed models provided fairly accurate prediction of hair greying but most of the prediction information was fulfilled by age itself. DNA variants were found to explain $<10 \%$ of hair greying variation and have small impact on prediction parameters thus confirming hair greying as being genetically a very complex trait. Although our study is a step forward in better understanding of greying processes, further studies, including 
genetic and molecular analyses are certainly needed. More statistical power is needed to identify additional markers of hair greying and to facilitate prediction of this trait especially in extreme age groups. The role of DNA methylation aging in hair greying development and its impact on prediction should also be evaluated. Finally, we propose a complex predictor that should include sex determination, genomic ancestry inference, analysis of DNA variation and epigenetic age estimation for a final prediction of hair greying status. The validity of this approach should be evaluated in the future studies.

\section{Methods}

\section{Sample collection and phenotypic classification}

Blood samples were collected from 998 unrelated individuals from Poland at age $\geq 18$ years (age range 19-62; Supplementary Table 1). Research participants were recruited at the Police Academy in Szczytno with financial support of the National Centre for Research and Development within the framework of the project NEXT (DOB-BIO7/17/01/2015). This research was approved by the Ethics Committee of the Jagiellonian University in Krakow (decision no. KBET/122/6120/11/2016). All participants provided written informed consent. With the help of the person conducting examination, participants completed a questionnaire including basic demographic data and phenotypic characteristics including hair greying status. Participants were asked to provide information on the occurrence of grey hair and the age at onset of hair greying. Additionally, high-quality photographs of the front part and the temporal part of the head were taken and evaluated for the progression of grey hair. 6stage classification of grey hair has been applied as the adaptation of the 5-stage classification system used by Adhikari and co-workers [17]. The following categories were distinguished: 1 - no greying; 2 - predominantly no greying, low number of single grey hair; 3 - higher number of single grey hair (all over the head); 4 - significant greying with patches of grey hair; 5 - predominant greying; 6 - totally white hair (Fig. 1). For the purpose of statistical calculations, a simplified 3-stage classification was used, where categories 2 and 3 were merged and accounted for 'mild greying' while categories 4, 5 and 6 were combined into a category 'severe greying'. Categories 'mild greying' and 'severe greying' were pooled for some analyses classifying hair greying as the binary outcome (greying vs. no greying). Participants were surveyed for hair dyeing, and if hair dyeing was found, detailed information on the condition and number of grey hair was collected.

\section{DNA extraction and quantification}

Whole blood samples collected from all volunteers were subjected to DNA extraction using PrepFiler ${ }^{\mathrm{Tm}}$ Forensic
DNA Extraction Kit (ThermoFisher Scientific) according to the manufacturer's protocol. DNA concentration was measured using Qubit dsDNA High-Sensitivity Assay Kit (Thermo Fisher Scientific) and Plexor ${ }^{\circ}$ HY System (Promega).

\section{Whole-exome sequencing}

WES analysis was conducted in a carefully selected cohort of 149 individuals maintaining adequate representativeness of particular phenotypic categories. Exonic regions (66 Mbp) have been enriched by regulatory sequences for $>160$ loci previously associated with human appearance traits (including pigmentation, hair loss, hair shape/thickness) (1.5 Mbp) extracted from Nencki Genomics Database and FANTOM [67-69]. Libraries were prepared using SeqCap EZ MedExome Target Enrichment Kit (Roche NimbleGen, Wisconsin, USA). Sequencing was conducted on HiSeq1500 Illumina machine offering economical and high-throughput sequencing. HiSeq SBS v4 chemistry and paired-end reads protocol were applied (Illumina, San Diego, CA USA). Raw data generated with WES were subjected to the bioinformatic pipeline aimed at analysis of SNP variation. First, read quality control was performed with FastQC (http://www. bioinformatics.babraham.ac.uk/projects/fastqc). Next, reads were mapped to the GRCh38 human genome with Bowtie 2 [70]. To avoid allele amplification bias in variant calling the Picard MarkDuplicates command was used for duplicate reads removal (http://broadinstitute. github.io/picard/). To detect and correct systematic errors in base quality scores recalibration was performed with Genome Analysis Toolkit (GATK) BaseRecalibrator [71, 72]. Dbsnp138 available from GATK resource bundle was used as known sites database. Default parameter values were used. Regions which required realignment were selected by GATK RealignerTargetCreator, and local realignment was performed with GATK IndelRealigner $[71,72]$. Variant calling restricted to the target region was performed with GATK Unified Genotyper. Filtering was performed with GATK VariantFiltration. To put the variant confidence QUAL score into perspective of the amount of coverage available variants with $\mathrm{QD}<2.0$ were filtered out. Also variants in which reads supporting the alternate allele had significantly lower mapping quality scores (MQRankSum < -12.5) than those supporting the reference allele were filtered out. Genotypes with genotype quality $<20$ and depth $<12$ were treated as missing. For the downstream statistical analyses we have selected variants with less than $20 \%$ of missing data and global minor allele frequency (MAF) $\geq 5 \%$.

\section{Candidate hair greying SNPs selection using exome-wide association testing}

Exome-wide data (over $77 \mathrm{~K}$ SNPs) generated for 149 discovery samples was subjected to association testing to 
select candidate DNA variants for hair greying. As testing single variables is still the most common method in association studies (mainly due to computational burden) [73], such approach was also applied in the current study. Exome-wide association analyses were conducted using logistic regression methods for hair greying status defined as greying vs. no greying (binomial logistic regression; BLR) or using 3-stage or 6-stage classifications (multinomial ordinal logistic regression; MLR3 and MLR6). Analyses were conducted with $\mathrm{R}$ v3.5.2 using 'ordinal' package. Analyses were adjusted for age, sex and hair colour (dark vs. light) and additive allele effect was assumed. SNPs with $P$-values smaller than $5 \times 10^{-4}$ were considered as significant. Exome-wide association testing results were visualized using Manhattan and QQ graphs (Supplementary Figs. 4-5) plotted using $R$ v3.5.2 and 'qqman' library. SNPs with $P$-values $<5 \times$ $10^{-4}$ were subjected to LD pruning which was conducted using PLINK 1.07. SNPs with independent effects $\left(\mathrm{r}^{2} \leq 0.7\right)$ were retained in each region reducing the initial list of 50 candidate hair greying SNPs to 34 DNA variants.

\section{Candidate hair greying SNPs selection using literature data}

It is anticipated that the genetics and biochemical pathways underlying different hair characteristics may be to some degree overlapping $[17,57,58]$. Adhikari et al. have shown correlations between different head hair features [17]. At least several genes (e.g. EDAR, WNT10A, IRF4, SUCNR5) have been proposed to influence more than one hair feature. Therefore, in the current study SNPs previously linked with hair loss, hair shape/thickness and pigmentation were evaluated for their association with hair greying. A detailed review of the literature allowed the selection of 344 SNPs (including 8 SNPs associated with hair greying, 90 SNPs associated with hair shape, 12 SNPs associated with hair thickness, 128 SNPs associated with hair loss (with 2 SNPs overlapping between hair shape and hair loss) and 108 SNPs associated with pigmentation) (Table 2 and Supplementary Table 5).

\section{Multiplex SNP genotyping using lon Torrent S5}

The final set of 378 candidate SNPs (including 34 WESselected variants and 344 literature based-selected variants) was genotyped in a replication cohort of 849 Polish samples. Targeted NGS implemented in Ion AmpliSeq ${ }^{\text {тм }}$ technology (ThermoFisher) which is offering efficient, sensitive and high-multiplexing solution was used to collect SNP genotypes. Data for the selected SNPs were generated within two Ion AmpliSeq ${ }^{\mathrm{Tm}}$ panels covering a total of 828 SNPs, including 378 candidate SNPs for head hair greying and 450 candidate SNPs for other human appearance traits studied in the project NEXT. Primer pools were designed using an Ion AmpliSeq ${ }^{\mathrm{Tm}}$ Designer tool (https://www.ampliseq.com) with a support of Thermo Fisher Scientific. Due to technical difficulties 3 SNPs: TRPC3 rs1396082, FBN3 rs56243829 and $D M K N$ rs79338830 were replaced by SNPs in LD (TRPC3 rs34306906, FBN3 rs72993531 and DMKN rs77995042). DNA libraries were prepared using the Ion AmpliSeq $^{\mathrm{TM}}$ Library Kit 2.0 according to the manufacturer's protocol with a slight modification of PCR step (5-10 ng of DNA in 5 or $10 \mu \mathrm{L}$ of total reaction volume). DNA libraries were quantified using Agilent High Sensitivity DNA Kit (Agilent Technologies, Santa Clara, USA) or Qubit dsDNA High-Sensitivity Assay Kit (Thermo Fisher Scientific), and then normalized to $40 \mathrm{pM}$. DNA libraries were combined in equal proportions and then template preparation was conducted using the Ion $520^{\mathrm{rm}}$ \& Ion $530^{\mathrm{mm}}$ Kit-Chef and the Ion Chef System. Sequencing was performed with the Ion $\mathrm{S}^{\mathrm{TM}}$ platform using Ion $530^{\mathrm{Tm}}$ Chips. Raw data were analysed using Torrent Server and SNPs were called using Torrent Variant Caller v5.6.0.4 or alternatively HID SNP Genotyper plugin v5.2.2. Missing SNP-data were at $0.1 \%$. The variable selection algorithms used in our research do not work on a set of data with missing values. Given the presence of missing data in neural network models, this would also result in poorer model quality. Therefore, the missing data were filled using 'missForest' method in R v3.5.2 (with a total number of trees equal to 500) and analyses were conducted on a more numerous data set.

\section{Association testing in a replication cohort}

The whole set of genotyped SNPs was subjected to association testing in a replication cohort of 849 samples. Single-SNP association analyses were carried out with BLR and MLR3/MLR6 using libraries under R v3.5.2. All the results were adjusted for age and sex (in case of pigmentation-linked SNPs adjustment for hair colour was also applied) and additive allele effect was assumed. Results with $P$-values $<0.05$ were considered as statistically significant. To visualize the effect of IRF4 rs12203592 on hair greying development CHAID (Chisquared Automatic Interaction Detection) analysis was conducted which is a classification method used to generate decision trees by using chi-square statistics. CHAI $\mathrm{D}$ tree was generated with PS IMAGO PRO 5.0 (IBM SPSS Statistics 25) for a 849-sample cohort using the data for IRF4 rs12203592 and age only.

\section{Pre-selection of SNPs for prediction model}

Methods allowing simultaneous analysis of all the tested SNPs are assumed to outperform single SNP-tests in a selection of a final set of predictors [32]. As traditional methods like regression may not be efficient when 
working with a large number of SNPs we used mRMRe feature selection approach (https://www.rdocumentation.org/ packages/mRMRe/versions/2.0.5) [74, 75]. mRMRe method is a fast framework for finding a set of the most relevant variables, based on a series of measures of relevance to the analysed trait and redundancy between the tested variables, thus outperforming classical approaches in terms of prediction accuracy [75]. mRMRe approach was applied to the genotypic data generated for all 378 candidate SNPs in a replication cohort, including information on age and sex. Analyses were conducted for hair greying defined in a binary way and using 3-stage classification. Classic mRMRe was applied and the top 30 variables in the output were generated. A set of 30 variables was analysed in terms of scoring and pruned based on analysis of scree plots (Supplementary Fig. 2). The resulting pre-selected set of predictors was further evaluated for the impact of particular markers on prediction accuracy measured by the area under the ROC curve (AUC) value as described in Section Prediction modelling using artificial $\mathrm{NN}$ and model validation.

\section{Prediction modelling using artificial NN and model validation}

Prediction modelling was conducted using artificial neural network (NN) approach and a set of 849 samples. We decided not to divide samples into separate groups used for markers pre-selection and model building in order not to reduce the size of the samples. Thus, the selection of final markers was treated as a part of the model training, as in the wraper approach. Two prediction models were developed allowing prediction of hair greying at the binary level; greying vs. no greying (BNN; binary neural network) and assuming three states of hair greying; no greying, mild greying and severe greying (MNN; multi-class neural network). Multilayer perceptron with one hidden layer and an automatically selected number of neurons was used. Details of the parameters of the method used can be found in our previous study [76]. The impact of particular markers included in the mRMRe-based pre-selected set of predictors on prediction accuracy was evaluated by determining the value of the AUC parameter each time next variable with the highest mRMRe score was incorporated into the model. The analysis was stopped and the list of predictors was pruned after no clear increase in AUC value was observed (Supplementary Fig. 1). Prediction performance of the final models was verified using 10-fold crossvalidation procedure, as described in detail in our previous work [76]. Then final prediction accuracy parameters including AUC, sensitivity, specificity, negative prediction value (NPV) and positive prediction value (PPV) [77] were calculated on the excluded $k$ th parts of the data using the pooling strategy [78]. Analyses were carried out using PS IMAGO PRO 5.0 (IBM SPSS Statistics 25).

\section{Population analyses}

Allele frequencies for the 13 SNPs included in the developed BNN and MNN models were plotted on the world map using allele frequency data extracted from "The 1000 Genomes Project" (https://www.ensembl.org/index. html) and using ArcMap 10.7 under ArcGIS Desktop software (Esri, Redlands, California). This data comes from 2504 subjects (19 worldwide countries) assigned to one of the following biogeographic ancestries: Europeans (EUR), Africans (AFR), admixed Americans (AMR), South Asians (SAS) and East Asians (EAS). In the next step the genotypes for these samples were extracted from http://grch37.ensembl.org/Homo_sapiens/Info/Index and analysed using BNN model. However, due to the lack of age and sex data for the samples, BNN model was rebuilt using DNA variants as the predictors only prior to analysis. Generated prediction probabilities for grey hair were then compared between populations.

\section{Supplementary information}

Supplementary information accompanies this paper at https://doi.org/10 1186/s12864-020-06926-y.

Additional file 1: Supplementary Fig. 1. The applied workflow for the final selection of predictors for head hair greying. Supplementary

Fig. 2. Scree plots generated for the top predictors selected with mRMRe method for A binary hair greying classification and B 3-stage hair greying classification. The mRMRe score values were plotted against their rank in the selected set of predictors. The suggestive cutoff point is indicated by the black horizontal line. In order to increase the transparency of the graphs and to improve determination of the cut-off point age (for binary hair greying classification) and age + sex (for 3-stage classification) were excluded when plotting graphs because of their significantly higher mRMRe scores. The order of the remaining predictors is in accordance with Supplementary Table 6. Supplementary Fig. 3. Global allele frequency distribution for 13 SNPs included in hair greying prediction models (A rs164741; B rs1005241; C rs1127228; D rs1683723; E rs2361506; F rs2416699; G rs2814331; H rs7680591; I rs10928235; J rs12203592; K rs45483393; L rs59733750; M rs68088846). Allele frequencies for the selected SNPs were plotted on the world map using data from "The 1000 Genomes Project" (http://grch37.ensembl.org/Homo_sapiens/Info/Index) and ArcMap 10.7 under ArcGIS Desktop software (Esri, Redlands, California). Supplementary Fig. 4. Manhattan plot of three EWAS analyses conducted in a 149 sample set for human head hair greying defined as A greying vs. no greying (BLR analysis); B no greying vs. mild greying vs. severe greying (MLR3 analysis); C 6-stage hair greying classification (MLR6 analysis). The -log10 (P-values) were plotted for each SNP under study according to its chromosomal position (GRCh38). The suggestive significance threshold ( -value $=5 \times 10-4)$ is indicated as a black horizontal line and SNPs that reached the suggestive significance threshold are marked with green. Supplementary Fig. 5. Q-Q plots of three EWAS analyses conducted in a 149 sample set for human head hair greying defined as A greying vs. no greying (BLR analysis); B no greying vs. mild greying vs. severe greying (MLR3 analysis); C 6-stage hair greying classification (MLR6 analysis). Supplementary Table 1. Characteristics of the study population including discovery and replication/prediction modelling cohorts. Supplementary Table 2. LD analysis conducted for exome-wide selected SNPS. Supplementary Table 3. A selection of exome-wide identified SNPs (P-value $<5 \times 10-4$ ) associated with hair greying in a discovery cohort of 149 individuals from Poland. Supplementary Table 4. Replication analysis of EWAS results in a replication cohort of 849 Polish individuals. Supplementary Table 5. Results of association testing for 336 literature-based selected candidate SNPs for hair greying associated previously with hair colour/pigmentation, hair loss, hair shape and hair 
thickness. Supplementary Table 6. The results of mRMRe analysis for binary and 3 -stage hair greying classification conducted in a 849-sample cohort

Additional file 2: Supplementary Table 7. The genotype-phenotype dataset generated with whole-exome sequencing.

Additional file 3: Supplementary Table 8. The genotype-phenotype dataset generated with targeted next-generation sequencing.

\section{Abbreviations}

AFR: Africans; AMR: Admixed Americans; AIM: Ancestry informative markers; AUC: Area under the ROC curve; BGA: Biogeographic ancestry; BLR: Binomial logistic regression; BNN: Binary neural network; CHAID: Chi-squared Automatic Interaction Detection; Cl: Confidence interval; CV: Cross-validation; DNA: Deoxyribonucleic acid; EAS: East Asians; EUR: Europeans; FGF: Fibroblast growth factor; GATK: Genome Analysis Toolkit; GWAS: Genome-wide association study; LD: Linkage disequilibrium; MAF: Minor allele frequency MLR: Multinomial ordinal logistic regression; MNN: Multi-class neural network; MPB: Male pattern baldness; mRMRe: The minimum redundancy maximum relevance; MSCs: Melanocyte stem cells; NGS: Next-generation sequencing; NN: Neural network; NPV: Negative prediction value; PCR: Polymerase Chain Reaction; PPV: Positive prediction value; SAS: South Asians; SNP: Single nucleotide polymorphism; STR: Short tandem repeat; TMEM: Transmembrane proteins; WES: Whole-exome sequencing

\section{Acknowledgements}

The authors would like to thank all sample donors for their contribution to this project.

\section{Authors' contributions}

$M S, W B, R P$ and TG received funding for the study. EP, MS, WB, RP, TG contributed to the study conception and design. MZ collected the samples and questionnaires and interpreted the phenotypic data. EP evaluated photographs. MD and RP were responsible for designing and conducting whole-exome sequencing experiments. PZ and EP conducted bioinformatic analyses of whole-exome sequencing data. MKB, AJ, AW and MB prepared libraries and carried out NGS runs. EP and MKB performed bioinformatic analysis of NGS targeted data. EP and JKP conducted statistical analyses. EP interpreted the final data and wrote the manuscript. All authors read and approved the final manuscript.

\section{Funding}

This work was supported by the grant from the National Centre for Research and Development in Poland [grant number DOB-BIO7/17/01/2015]. EP and WB received support from the European Union's Horizon 2020 Research and Innovation Programme under grant agreement No. 740580 within the framework of the Visible Attributes through Genomics (VISAGE) Project and Consortium. The funding bodies had no role in study design, analysis and interpretation of data or preparation of the manuscript. The open-access publication of this article was funded by the Priority Research Area BioS under the program "Excellence Initiative - Research University" at the JagielIonian University in Krakow.

\section{Availability of data and materials}

The genotype-phenotype datasets generated with WES and targeted nextgeneration sequencing are included within the article as Supplementary Table 7 and Supplementary Table 8, respectively.

\section{Ethics approval and consent to participate}

The study was approved by the Ethics Committee of the Jagiellonian University in Krakow (decision no. KBET/122/6120/11/2016). All participants provided written informed consent.

\section{Consent for publication}

Not applicable.

\section{Competing interests}

The authors declare that they have no competing interests.

\section{Author details}

Malopolska Centre of Biotechnology, Jagiellonian University, Kraków, Poland. ${ }^{2}$ Faculty of Biochemistry, Biophysics and Biotechnology, Jagiellonian University, Kraków, Poland. ${ }^{3}$ Faculty of Mathematics and Computer Science, Nicolaus Copernicus University, Toruń, Poland. ${ }^{4}$ Institute of Environmental Sciences, Faculty of Biology, Jagiellonian University, Kraków, Poland. ${ }^{5}$ Central Forensic Laboratory of the Police, Warsaw, Poland. 'Laboratory of Bioinformatics, Nencki Institute of Experimental Biology, Warsaw, Poland.

${ }^{7}$ Faculty of Law and Administration, Department of Criminology and Forensic Sciences, University of Warmia and Mazury in Olsztyn, Olsztyn, Poland. ${ }^{8}$ Department of Forensic Medicine, Collegium Medicum of the Nicolaus Copernicus University, Bydgoszcz, Poland. ${ }^{9}$ Department of Medical Genetics, Warsaw Medical University, Warsaw, Poland.

Received: 19 March 2020 Accepted: 20 July 2020

Published online: 05 August 2020

\section{References}

1. Christensen K, Thinggaard M, McGue M, Rexbye H, Hjelmborg JV, Aviv A, et al. Perceived age as clinically useful biomarker of ageing: cohort study. BMJ. 2009;339:b5262

2. Gunn DA, Rexbye H, Griffiths CE, Murray PG, Fereday A, Catt SD, et al. Why some women look young for their age. PLoS One. 2009;4:e8021.

3. Gunn DA, Larsen LA, Lall JS, Rexbye H, Christensen K. Mortality is written on the face. J Gerontol A Biol Sci Med Sci. 2016;71:72-7.

4. Vierkötter A, Ranft U, Krämer U, Sugiri D, Reimann V, Krutmann J. The SCIN EXA: a novel, validated score to simultaneously assess and differentiate between intrinsic and extrinsic skin ageing. J Dermatol Sci. 2009;53:207-11.

5. Seiberg M. Age-induced hair greying - the multiple effects of oxidative stress. Int J Cosmet Sci. 2013;35:32-8.

6. Kayser M. Forensic DNA phenotyping: predicting human appearance from crime scene material for investigative purposes. Forensic Sci Int Genet. 2015:18:33-48.

7. Keogh EV, Walsh RJ. Rate of greying of human hair. Nature. 1965;207:877-8.

8. Tobin DJ, Paus R. Graying: gerontobiology of the hair follicle pigmentary unit. Exp Gerontol. 2001;36:29-54

9. Jo SK, Lee JY, Lee Y, Kim CD, Lee JH, Lee YH. Three streams for the mechanism of hair graying. Ann Dermatol. 2018;30:397-401.

10. Neste DV, Tobin DJ. Hair cycle and hair pigmentation: dynamic interactions and changes associated with aging. Micron. 2004;35:193-200.

11. Panhard S, Lozano I, Loussouarn G. Greying of the human hair: a worldwide survey, revisiting the '50' rule of thumb. Br J Dermatol. 2012;167:865-73.

12. Nishimura EK, Granter SR, Fisher DE. Mechanisms of hair graying: incomplete melanocyte stem cell maintenance in the niche. Science. 2005;307:720-4.

13. Jadkauskaite $L$, Coulombe PA, Schäfer M, Dinkova-Kostova AT, Paus R, Haslam IS. Oxidative stress management in the hair follicle: could targeting NRF2 counter age-related hair disorders and beyond? Bioessays. 2017;39.

14. Arck PC, Overall R, Spatz K, Liezman C, Handjiski B, Klapp BF, et al. Towards a "free radical theory of graying": melanocyte apoptosis in the aging human hair follicle is an indicator of oxidative stress induced tissue damage. FASEB J. 2006:20:1567-9.

15. Choi HI, Choi Gl, Kim EK, Choi YJ, Sohn KC, Lee Y, et al. Hair greying is associated with active hair growth. Br J Dermatol. 2011;165:1183-9.

16. Shin H, Ryu HH, Yoon J, Jo S, Jang S, Choi M, et al. Association of premature hair graying with family history, smoking, and obesity: a cross-sectional study. J Am Acad Dermatol. 2015;72:321-7.

17. Adhikari K, Fontanil T, Cal S, Mendoza-Revilla J, Fuentes-Guajardo M, Chacón-Duque JC, et al. A genome-wide association scan in admixed Latin Americans identifies loci influencing facial and scalp hair features. Nat Commun. 2016;7:10815.

18. Weissbrod O, Flint J, Rosset S. Estimating SNP-based heritability and genetic correlation in case-control studies directly and with summary statistics. Am J Hum Genet. 2018;103:89-99.

19. Han J, Kraft P, Nan H, Guo Q, Chen C, Qureshi A, et al. A genome-wide association study identifies novel alleles associated with hair color and skin pigmentation. PLoS Genet. 2008:4:e1000074

20. Jacobs LC, Hamer MA, Gunn DA, Deelen J, Lall JS, van Heemst D, et al. A genomewide association study identifies the skin color genes IRF4, MC1R, ASIP, and BNC2 influencing facial pigmented spots. J Invest Dermatol. 2015;135:1735-42. 
21. Hagenaars SP, Hill WD, Harris SE, Ritchie SJ, Davies G, Liewald DC, et al. Genetic prediction of male pattern baldness. PLoS Genet. 2017;13:e1006594

22. Kukla-Bartoszek M, Pośpiech E, Woźniak A, Boroń M, Karłowska-Pik J, Teisseyre $P$, et al. DNA-based predictive models for the presence of freckles. Forensic Sci Int Genet. 2019;42:252-9.

23. Praetorius C, Grill C, Stacey SN, Metcalf AM, Gorkin DU, Robinson KC, et al. A polymorphism in IRF4 affects human pigmentation through a tyrosinasedependent MITF/TFAP2A pathway. Cell. 2013;155:1022-33.

24. McGill GG, Horstmann M, Widlund HR, Du J, Motyckova G, Nishimura EK, et al. Bcl2 regulation by the melanocyte master regulator mitf modulates lineage survival and melanoma cell viability. Cell. 2002;109:707-18.

25. Dawber RP. Integumentary associations of pernicious anaemia. $\mathrm{Br} \mathrm{J}$ Dermatol. 1970;82:221-3.

26. Blumen SC, Bevan S, Abu-Mouch S, Negus D, Kahana M, Inzelberg R, et al. A locus for complicated hereditary spastic paraplegia maps to chromosome 1q24-q32. Ann Neurol. 2003;54:796-803.

27. Domínguez-Gerpe L, Araújo-Vilar D. Prematurely aged children: molecular alterations leading to Hutchinson-Gilford progeria and Werner syndromes. Curr Aging Sci. 2008;1:202-12.

28. Pingault V, Ente D, Dastot-Le Moal F, Goossens M, Marlin S, Bondurand N. Review and update of mutations causing Waardenburg syndrome. Hum Mutat. 2010;31:1-16.

29. Ritchie MD. Finding the epistasis needles in the genome-wide haystack. Methods Mol Biol. 2015;1253:19-33

30. Kim H, Grueneberg A, Vazquez Al, Hsu S, de Los Campos G. Will big data close the missing heritability gap? Genetics. 2017;207:1135-45.

31. de Los CG, Vazquez Al, Hsu S, Lello L. Complex-trait prediction in the era of big data. Trends Genet. 2018;34:746-54

32. Hoggart CJ, Whittaker JC, De lorio M, Balding DJ. Simultaneous analysis of all SNPs in genome-wide and re-sequencing association studies. PLOS Genet. 2008;4:e1000130.

33. Schnohr P, Lange P, Nyboe J, Appleyard M, Jensen G. Gray hair, baldness, and wrinkles in relation to myocardial infarction: the Copenhagen city heart study. Am Heart J. 1995;130:1003-10.

34. Orr-Walker BJ, Evans MC, Ames RW, Clearwater JM, Reid IR. Premature hair graying and bone mineral density. J Clin Endocrinol Metab. 1997;82:3580-3.

35. Kocaman SA, Cetin M, Durakoglugil ME, Erdoğan T, Çanga A, Çiçek Y, et al. The degree of premature hair graying as an independent risk marker for coronary artery disease: a predictor of biological age rather than chronological age. Anadolu Kardiyol Derg. 2012;12:457-63.

36. Harris ML, Fufa TD, Palmer JW, Joshi SS, Larson DM, Incao A, et al. A direct link between MITF, innate immunity, and hair graying. PLoS Biol. 2018;16: e2003648.

37. Yashin Al, Wu D, Arbeev KG, Ukraintseva SV. Joint influence of smalleffect genetic variants on human longevity. Aging (Albany NY). 2010;2: 612-20.

38. Law MH, Medland SE, Zhu G, Yazar S, Viñuela A, Wallace L, et al. Genomewide association shows that pigmentation genes play a role in skin aging. J Invest Dermatol. 2017:137:1887-94.

39. Zhang Q, Marioni RE, Robinson MR, Higham J, Sproul D, Wray NR. Genotype effects contribute to variation in longitudinal methylome patterns in older people. Genome Med. 2018;10:75.

40. Bandyopadhyay D, Medrano EE. The emerging role of epigenetics in cellula and organismal aging. Exp Gerontol. 2003;38:1299-307.

41. Hunt SE, McLaren W, Gil L, Thormann A, Schuilenburg H, Sheppard D, et al. Ensembl variation resources. Database. 2018. https://doi.org/10.1093/ database/bay 119.

42. Riviere JB, Ramalingam S, Lavastre V, Shekarabi M, Holbert MS, Lafontaine J, et al. KIF1A, an axonal transporter of synaptic vesicles, is mutated in hereditary sensory and autonomic neuropathy type 2. Am J Hum Genet. 2011;89:219-30.

43. Erlich Y, Edvardson S, Hodges E, Zenvirt S, Thekkat P, Shaag A, et al. Exome sequencing and disease-network analysis of a single family implicate a mutation in KIF1A in hereditary spastic paraparesis. Genome Res. 2011;21: 658-64

44. Lee JYW, Hsu CK, Michael M, Nanda A, Liu L, McMillan J, et al. Large intragenic deletion in DSTYK underlies autosomal-recessive complicated spastic paraparesis, SPG23. Am J Hum Genet. 2017;100:364-70.

45. Baptista Fl, Pinto MJ, Elvas F, Almeida RD, Ambrósio AF. Diabetes alters KIF1A and KIF5B motor proteins in the hippocampus. PLoS One. 2013;8: e65515
46. Miranda JJ, Taype-Rondan A, Tapia JC, Gastanadui-Gonzalez MG, RomanCarpio R. Hair follicle characteristics as early marker of type 2 diabetes. Med Hypotheses. 2016;95:39-44.

47. Feldman EL, Callaghan BC, Pop-Busui R, Zochodne DW, Wright DE, Bennett DL, et al. Diabetic neuropathy. Nat Rev Dis Primers. 2019;5:41.

48. Fang S, Han J, Zhang M, Wang L, Wei Q, Amos Cl, et al. Joint effect of multiple common SNPs predicts melanoma susceptibility. PLoS One. 2013;8: e85642.

49. Fujioka Y, Kimata Y, Nomaguchi K, Watanabe K, Kohno K. Identification of a novel non-structural maintenance of chromosomes (SMC) component of the SMC5-SMC6 complex involved in DNA repair. J Biol Chem. 2002;277: 21585-91.

50. Negishi-Koga T, Shinohara M, Komatsu N, Bito H, Kodama T, Friedel R, et al. Suppression of bone formation by osteoclastic expression of semaphorin 4D. Nature Med. 2011;17:1473-80.

51. Schmit K, Michiels C. TMEM proteins in Cancer: a review. Front Pharmacol 2018;9:1345

52. Medland SE, Nyholt DR, Painter JN, McEvoy BP, McRae AF, Zhu G, et al. Common variants in the trichohyalin gene are associated with straight hair in Europeans. Am J Hum Genet. 2009;85:750-5.

53. Heilmann-Heimbach S, Herold C, Hochfeld LM, Hillmer AM, Nyholt DR, Hecker J, et al. Meta-analysis identifies novel risk loci and yields systematic insights into the biology of male-pattern baldness. Nat Commun. 2017;8: 14694.

54. Pirastu N, Joshi PK, de Vries PS, Cornelis MC, McKeique PM, Keum N, et al. GWAS for male-pattern baldness identifies 71 susceptibility loci explaining $38 \%$ of the risk. Nat Commun. 2017;8:1584.

55. Higgins CA, Petukhova L, Harel S, Ho YY, Drill E, Shapiro L, et al. FGF5 is a crucial regulator of hair length in humans. Proc Nat Acad Sci. 2014;111: 10648-53.

56. Branicki W, Liu F, van Duijn K, Draus-Barini J, Pośpiech E, Walsh S, et al. Model-based prediction of human hair color using DNA variants. Hum Genet. 2011;129:443-54

57. Marcińska M, Pośpiech E, Abidi S, Dyrberg JA, van den Berge M, Carracedo Á, et al. Evaluation of DNA variants associated with androgenetic alopecia and their potential to predict male pattern baldness. PLoS One. 2015;10: e0127852.

58. Pośpiech E, Karłowska-Pik J, Marcińska M, Abidi S, Andersen JD, Berge MVD, et al. Evaluation of the predictive capacity of DNA variants associated with straight hair in Europeans. Forensic Sci Int Genet. 2015;19:280-8.

59. Liu F, Hamer MA, Heilmann S, Herold C, Moebus S, Hofman A, et al. Prediction of male pattern baldness from genotypes. Eur J Hum Genet. 2016;24:895-902.

60. Pośpiech E, Chen Y, Kukla-Bartoszek M, Breslin K, Aliferi A, Andersen JD, et al Towards broadening forensic DNA Phenotyping beyond pigmentation: improving the prediction of head hair shape from DNA. Forensic Sci Int Genet. 2018;37:241-51.

61. Horvath S. DNA methylation age of human tissues and cell types. Genome Biol. 2013:14:R115.

62. Hannum G, Guinney J, Zhao L, Zhang L, Hughes G, Sadda S, et al. Genomewide methylation profiles reveal quantitative views of human aging rates. Mol Cell. 2013;49:359-67.

63. Lu AT. Xue L, Salfati EL, Chen BH, Ferrucci L, Levy D, et al. GWAS of epigenetic aging rates in blood reveals a critical role for TERT Nat Commun. 2018;9:387.

64. Hillmer A, Freudenberg J, Myles S, Herms S, Tang K, Hughes DA, et al. Recent positive selection of a human androgen receptor/ectodysplasin A2 receptor haplotype and its relationship to male pattern baldness. Hum Genet. 2009;126:255-64.

65. Claes $\mathrm{P}$, Hill H, Shriver MD. Toward DNA-based facial composites: preliminary results and validation. Forensic Sci Int Genet. 2014;13:208-16.

66. Schneider P, Prainsack B, Kayser M. The Use of Forensic DNA Phenotyping in Predicting Appearance and Biogeographic Ancestry. Dtsch Arztebl Int. 2019:51-52:873-880

67. Krystkowiak I, Lenart J, Debski K, Kuterba P, Petas M, Kaminska B, et al. Nencki Genomics Database--Ensembl Funcgen Enhanced with Intersections, User Data and Genome-Wide TFBS Motifs. Database. 2013;bat069 https://doi.org/10.1093/database/bat069.

68. Andersson R, Gebhard C, Miguel-Escalada I, Hoof I, Bornholdt J, Boyd M, et al. An atlas of active enhancers across human cell types and tissues. Nature. 2014;507:455-61. 
69. FANTOM. Consortium and the RIKEN PMI and CLST (DGT), Forrest AR, Kawaji $\mathrm{H}$, Rehli M, Baillie JK, de Hoon MJ, et al. A Promoter-Level Mammalian Expression Atlas Nature. 2014;507:462-70.

70. Langmead B, Trapnell C, Pop M, Salzberg SL. Ultrafast and memory-efficient alignment of short DNA sequences to the human genome. Genome Biol. 2009;10:R25.

71. McKenna A, Hanna M, Banks E, Sivachenko A, Cibulskis K, Kernytsky A, et al. The genome analysis toolkit: a MapReduce framework for analyzing nextgeneration DNA sequencing data. Genome Res. 2010;20:1297-303.

72. Depristo MA, Banks E, Poplin R, Garimella KV, Maguire JR, Hartl C, et al. A framework for variation discovery and genotyping using next-generation DNA sequencing data. Nat Genet. 2011;43:491-501.

73. Frommlet F, Bigdan M, Ramsey D. Phenotypes and genotypes. SpringerVerlag, London: The Search for Influential Genes; 2016.

74. Ding C, Peng H. Minimum redundancy feature selection from microarray gene expression data. J Bioinforma Comput Biol. 2005;3:185-205.

75. De Jay N, Papillon-Cavanagh S, Olsen C, El-Hachem N, Bontempi G, HaibeKains B. mRMRe: an $\mathrm{R}$ package for parallelized mRMR ensemble feature selection. Bioinformatics. 2013;29:2365-8.

76. Pośpiech E, Karłowska-Pik J, Ziemkiewicz B, Kukla M, Skowron M, Wojas-Pelc $A$, et al. Further evidence for population specific differences in the effect of DNA markers and gender on eye colour prediction in forensics. Int J Legal Med. 2016;130:923-34

77. Pośpiech E, Draus-Barini J, Kupiec T, Wojas-Pelc A, Branicki W. Prediction of eye color from genetic data using Bayesian approach. J Forensic Sci. 2012; 57:880-6.

78. Parker BJ, Günter S, Bedo J. Stratification bias in low signal microarray studies. BMC Bioinformatics. 2007:8:326.

\section{Publisher's Note}

Springer Nature remains neutral with regard to jurisdictional claims in published maps and institutional affiliations.

Ready to submit your research? Choose BMC and benefit from:

- fast, convenient online submission

- thorough peer review by experienced researchers in your field

- rapid publication on acceptance

- support for research data, including large and complex data types

- gold Open Access which fosters wider collaboration and increased citations

- maximum visibility for your research: over $100 \mathrm{M}$ website views per year

At $\mathrm{BMC}$, research is always in progress.

Learn more biomedcentral.com/submissions 